\title{
Electronic Structure and Chemistry of Nanomaterials Embedded in a Matrix Using Atomically Resolved Near-edge Structures: The Example of Ferromagnetic Ni Nanowires Grown in $\mathrm{SrTiO}_{3}$
}

Matthieu Bugnet ${ }^{1}$, Quentin Ramasse ${ }^{2}$, Dominique Demaille ${ }^{3}$, Marcel Hennes ${ }^{3}$, Xiaorong Weng ${ }^{3}$, Yunlin Zheng $^{3}$, Franck Vidal ${ }^{3}$ and Guillaume Radtke ${ }^{3}$

${ }^{1}$ SuperSTEM Laboratory and School of Physics and School of Chemical and Process Engineering University of Leeds and CNRS MATEIS, VIlleurbanne, Rhone-Alpes, France, ${ }^{2}$ SuperSTEM Laboratory and University of Leeds, Daresbury, England, United Kingdom, ${ }^{3}$ Sorbonne Université - CNRS, Paris, Ilede-France, France

Electron energy-loss spectroscopy (EELS) in the aberration-corrected scanning transmission electron microscope (ac-STEM) is a widely used technique to achieve chemical mapping at the resolution of a single atomic-column. Beyond the purely elemental information, core-level excitation in EELS is a direct probe of the unoccupied site- and momentum- projected density of states. Such information about the electronic structure are reflected into the spectral fine structures above the Fermi level, the energy-loss near edge structures (ELNES), and provide a wealth of information to understand the structure and chemistry interplay in e.g. thin films [1], nanostructured bulk materials [2], and composite systems with embedded nanomaterials $[3,4]$. With a monochromator, the large majority of spectral features in core level excitation are resolved at and above the edge onset. More specifically, monochromated absorption edges reveal subtle information in the electronic structure of the element probed, which can then be linked to structural characteristics of the material $[5,6]$.

Overcoming the limits of current data storage technologies has become a major societal challenge. One of the proposed solutions consists in the growth of three dimensional spintronic structures by physical deposition methods. For instance, pulsed laser deposition of self-assembled ferromagnetic nanowires (NWs) embedded in, and epitaxially coupled to, an insulating matrix has recently attracted considerable attention from its peculiar magnetic anisotropy. The latter has been attributed to an efficient magnetoelastic coupling arising from epitaxial strain at the vertical interfaces between the nanowires and the matrix [7]. Despite the fact that the physical properties of these so-called vertically aligned nanostructures (VANs) are intimately governed by the vertical nanowire-matrix interface, studies addressing their structure/composition at the atomic scale are scarce, possibly due to the difficulty to access the interface. In this work, we highlight the contribution of monochromated core-loss EELS combined with ac-STEM to the understanding of chemical, structural and physical properties of a VAN, which consists of ferromagnetic nanowires, e.g. Ni, embedded in an insulating matrix, e.g. $\mathrm{SrTiO}_{3}$, as shown in Figure 1.

To study the $\mathrm{Ni}: \mathrm{SrTiO}_{3}$ interface, TEM thin foils were prepared in two geometries. For plane views, the back side of the sample was first mechanically thinned down to a thickness less than a few tens of micrometers. Then, argon ion milling was used in order to reach electron transparency. The same approach was employed for cross-sectional views. High energy resolution for ELNES $(\sim 160 \mathrm{meV})$ at $\sim 1 \AA$ spatial resolution was achieved in a monochromated Cs-corrected Nion HERMES dedicated STEM instrument, operating at $60 \mathrm{kV}$. 
The potential oxidation of nickel, via the formation of an $\mathrm{NiO}$ layer at the $\mathrm{Ni}: \mathrm{SrTiO}_{3}$ interface, is one of the major points of concerns for the prospect of controlling the magnetic properties of this heterostructure. In this regard, the Ni-L23 ELNES, which shows substantial differences between metallic $\mathrm{Ni}$ and $\mathrm{Ni}^{2+}$, as well as the $\mathrm{O}-\mathrm{K}$ and $\mathrm{Ti}-\mathrm{L}_{23}$ fine structures were investigated at the structurally abrupt $\mathrm{Ni}: \mathrm{SrTiO}_{3}$ interface [4]. This approach revealed the presence of a weak chemical interdiffusion over a few atomic planes, leading essentially to a metallization of the last $\mathrm{TiO}_{\mathrm{x}}(\mathrm{x}<2)$ atomic plane of the matrix, and to the formation of non-stoichiometric mixed Ni-Ti oxide on the external part of the NW. These results demonstrate the absence of $\mathrm{NiO}$, and highlight that $\mathrm{Ni}_{\mathrm{SrTiO}}$ is a model system for strain engineering studies of the magnetic anisotropy in vertically aligned nanocomposites. The effect of the vertical interface on the local electronic structure is further explored through variations of the Ti-L23 ELNES at the length scale of a single atomic column to the next, as shown in Figure 2.

This work highlights some of the advantages and limitations of combining atomic-resolution Z-contrast chemical imaging with elemental EELS mapping and fine structure information to better understand the structural and chemical nature of sharp interfaces in $\mathrm{Ni}_{\mathrm{SrTiO}}$. The approach described in this study could be applied to a wide range of materials, specifically complex systems such as heterostructures, and nanomaterials embedded in devices [8].
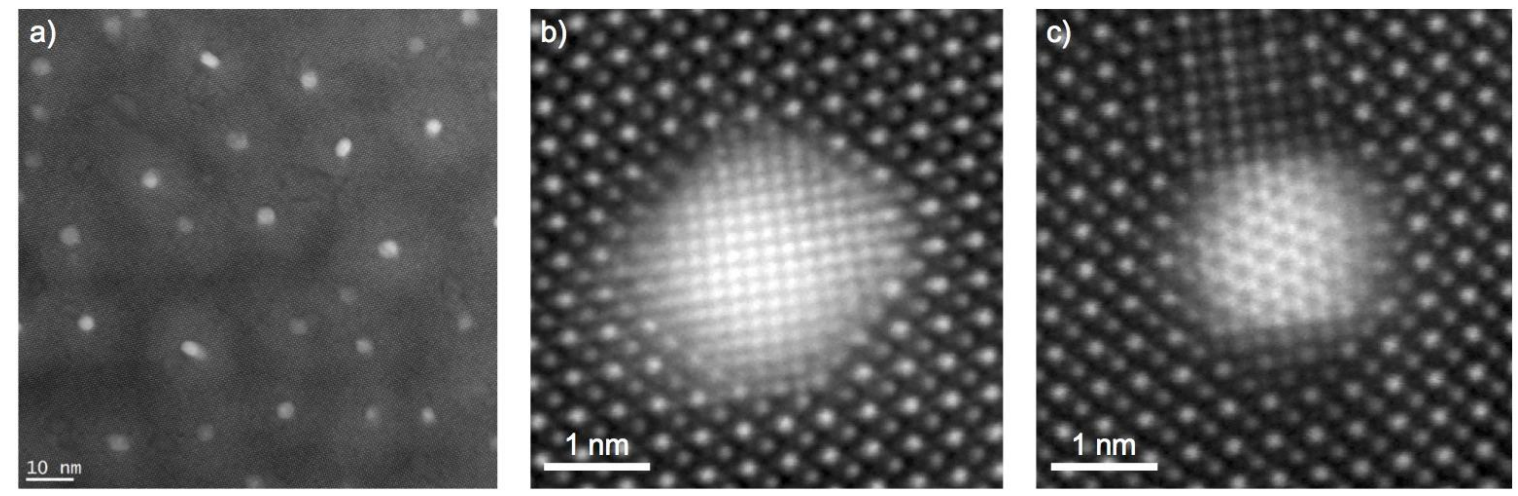

Figure 1. a) HAADF-STEM plane view of a Ni:SrTiO3/SrTiO3 vertically aligned nanostructures, where Ni NWs are epitaxially grown on SrTiO3(001) substrate, and embedded within an SrTiO3 matrix. High resolution HAADF-STEM images reveal the presence of both cubic (b), and hexagonal (c) epitaxiallygrown Ni nanowires.
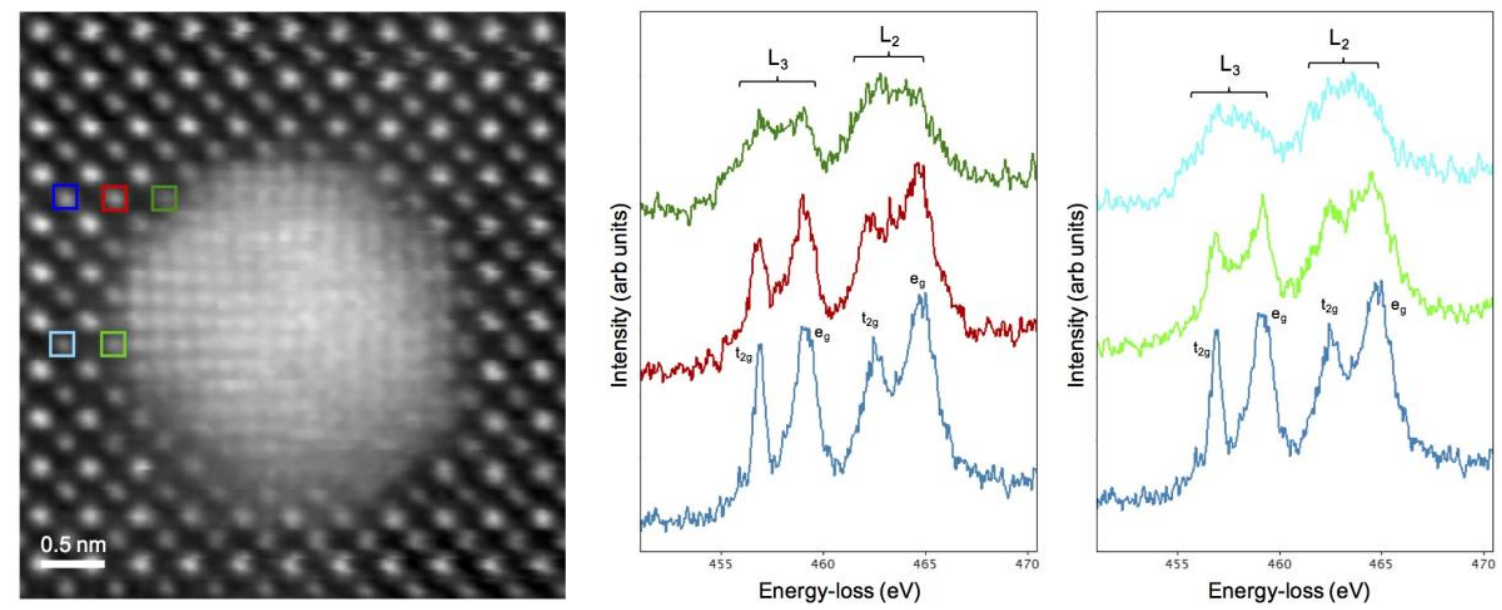
Figure 2. Variations of the Ti-L23 ELNES as a function of beam position at Ni:SrTiO3 interfaces with (001)Ni\|(001)SrTiO3 and [100]Ni\|[100]SrTiO3 epitaxial relationship. The octahedral t2g-eg crystal field splitting is indicated as a reference from the position furthest away from the interface (dark blue square).

References

[1] A. Teurtrie et al., Advanced Functional Materials, 29, 1904958 (2019).

[2] M. Bugnet et al., Science Advances, 2, e1501652 (2016).

[3] D. Zhou et al., Advanced Materials Interfaces, 2, 1500377 (2015).

[4] G. Radtke et al, Advanced Materials Interfaces, 6, 1900549 (2019).

[5] S. Lazar et al., Ultramicroscopy, 98, 249-257 (2004).

[6] M. Bugnet et al., Physical Review B, 93, 020102 (2016).

[7] V. Schuler et al., Nanotechnology, 27, 495601 (2016).

[8] STEM-EELS experiments were carried out at SuperSTEM, the EPSRC National Research Facility for Advanced Electron Microscopy. M. Bugnet is grateful to SuperSTEM for access to instruments, and to the University of Leeds for an Associate Visiting Professorship position. This work was supported by French state funds managed by the ANR within the Investissements d'Avenir programme (ANR-11IDEX-0004-02), within the framework of the Cluster of Excellence MATISSE led by Sorbonne Universités. 